\title{
Comparative Study between the Use of Room Temperature and Cold Intra-Thecal Heavy Bupivacaine and Its Effect on Intraoperative Shivering in Lower Limb Orthopedic Surgery
}

\author{
Hassanein Abd- Elkareem Hamzawy, Alsayed Mostafa Stohy, Wael Mohamed El-Mahdi, \\ Ayman Baligh Hassan Gamal* \\ Department of Anesthesia and Intensive Care, Faculty of Medicine, Al-Azhar University \\ *Corresponding author: Ayman Baligh Hassan Gamal, E-Mail: hossamesmail31@ gmail.com
}

\begin{abstract}
Background: the mechanisms responsible for shivering in patients undergoing surgery are intraoperative temperature loss increased sympathetic tone, pain, and systemic release of pyrogens, and the direct effect of local anesthetic temperature on temperature-sensitive neurons in the spinal cord.

Objective: to compare the effects of warm intrathecal bupivacaine versus cold intra-thecal bupivacaine on shivering and haemodynamic stability in parturient candidate for elective lower limb orthopedic surgery under spinal anesthesia.

Patients and Methods: the current study was carried out in in Alexandria Police hospital, on 80 parturient, aged 20-60 years old, ASA I-II, scheduled for elective lower limb orthopedic surgery under spinal anesthesia, after the approval from the Local Ethical Committee and informed written consents from all the patients of the study. Patients were randomly allocated into two equal groups (40 patients each); group R (Warm group), group C (Cold group).

Results: Comparison between the two studied groups showed no significant statistical differences at all the measured times.

Conclusion: Shivering continues to be a common problem after spinal anesthesia. The etiology of this symptom is unknown, and there is no definite treatment.
\end{abstract}

Keywords: Cerebral spinal fluid, General anaesthesia, Intravenous

\section{INTRODUCTION}

Shivering associated with spinal anesthesia is an extremely frequently complication that occurs in up to $55 \%$ of the patients ${ }^{(\mathbf{1})}$. In addition to disrupting the comfort of the patient, shivering has various hemodynamic effects, including increased oxygen consumption, increased carbon dioxide production and increased cardiac effort ${ }^{(2)}$. Also shivering may interfere with the monitoring of ECG, blood pressure and oxygen saturation ${ }^{(3)}$.

The mechanisms responsible for shivering in patients undergoing surgery are intra-operative temperature loss increased sympathetic tone, pain, and systemic release of pyrogens, and the direct effect of local anesthetic temperature on temperaturesensitive neurons in the spinal $\operatorname{cord}^{(4)}$.

The central nervous system (CNS), including the spinal cord, receives thermal signals from the body and plays an essential role in the regulation and maintenance of body temperature ${ }^{(3)}$.
Several factors affect the dissemination of the local anesthetic agents (LA) used in spinal anaesthesia within cerebral spinal fluid (CSF). The density of the local anaesthetic used plays a significant role in the dissemination within the CSF and the level and duration of the effect of spinal anaesthesia ${ }^{(6)}$.

Although many studies have been undertaken to treat shivering after spinal anesthesia by combined of warm parenteral fluids and warm local anesthetics significantly reduced the incidence of shivering, Some researchers have studied the effects of local anesthetic temperature after injection intrathecally on shivering ${ }^{(4)}$. The result of another study suggested the existence of thermosensory mechanisms in the human spinal canal and the effect of warming anesthetic solutions prior to injection to reduce the incidence of shivering ${ }^{(5)}$.

Orthopedic Surgery is a common surgical procedure concerned with injuries and conditions that affect the musculoskeletal 
system. It involves the upper and lower limbs and there is an increasing trend in the use of regional techniques for them ${ }^{(7)}$.

In modern anaesthesia practice, regional techniques are preferred to GA. These benefits include superior intraoperative pain control, attenuation of the surgical stress response, minimal systemic impairment, reduced blood loss and transfusion requirements, less postoperative nausea and vomiting, excellent postoperative analgesia, increased level of alertness after surgery, decreased hospitalization and $\operatorname{cost}^{(8)}$.

\section{AIM OF THE WORK}

The aim of this study is to compare the effects of room temperature intra-thecal heavy bupivacaine stored at $23^{\circ} \mathrm{C}$ and cold intra-thecal heavy bupivacaine stored at $6^{\circ} \mathrm{C}$ on shivering and haemodynamic stability during lower limb orthopedic surgery.

\section{PATIENTS AND METHODS Patients}

This study was carried in Alexandria Police hospital on 80 patients.

Study design: Patients were randomly assigned (double-blind, envelope randomization) into two equal groups (40 patients each) according to the local anesthetic temperature.

Inclusion criteria: Age between 20 and 60 years, patients of both sexes, ASA class I or II, and patient scheduled for elective lower limb orthopedic surgery.

Exclusion criteria: Any contraindications of spinal anesthesia as: Patient's refusal. Coagulation disorders or ongoing anticoagulation treatment. Severe aortic or mitral stenosis. Hypersensitivity to local anesthetics of the amide type. Active disease of the central nervous system such as meningitis, poliomyelitis, intracranial hemorrhage, sub-acute combined degeneration of the cord due to pernicious anemia and cerebral and spinal tumours. Spinal stenosis and active disease (e.g. spondylitis, tuberculosis, tumour) or recent trauma (e.g. fracture) in the vertebral column. Septicaemia. Pyogenic infection of the skin at or adjacent to the site of lumbar puncture. Cardiogenic or hypovolaemic shock $^{(9)}$. Patients with any neurological disease. Any patients ASA class III or IV.

Ethical considerations: Study procedures were reviewed and approved by Al-
Azhar University Research Ethics Committee. Study objectives and expected outcomes were explained to study participants. Participation in the study was voluntary. Confidentiality of data collected was ensured.

Intra-thecal anesthesia should only be undertaken by clinicians with the necessary knowledge and experience ${ }^{(10)}$.

Regional anesthetic procedures should always be performed in a properly equipped and staffed area. Resuscitative equipment and drugs should be immediately available and the anesthetist should remain in constant attendance $^{(10)}$.

The clinician responsible should take the necessary precautions to avoid intravascular injection and be appropriately trained and familiar with the diagnosis and treatment of side effects, systemic toxicity and other complications. If signs of acute systemic toxicity or total spinal block appear, injection of the local anesthetic should be stopped immediately ${ }^{(9)}$.

Like all local anesthetic drugs, bupivacaine may cause acute toxicity effects on the central nervous and cardiovascular systems, if utilized for local anesthetic procedures resulting in high blood concentrations of the drug. This is especially the case after unintentional intravascular administration or injection into highly vascular areas ${ }^{(9)}$.

\section{Methods}

Patients were randomly separated into 2 groups, forty patients in each group receive either heavy bupivacaine $0.5 \%$ stored at room temperature ( $\mathrm{R}$ group) or heavy bupivacaine $0.5 \%$ stored at $6^{\circ} \mathrm{C}$ (C group).

Group R (room temperature group): The case group will receive $20 \mathrm{mg}$ of heavy bupivacaine $0.5 \%$ stored at room temperature $\left(23^{\circ} \mathrm{C}\right)$ plus $25 \mu \mathrm{g}$ of fentanyl intra-thecally. Group C (cold group): The standard group will receive $20 \mathrm{mg}$ of heavy bupivacaine $0.5 \%$ stored at $6^{\circ} \mathrm{C}$ plus $25 \mu \mathrm{g}$ of fentanyl intrathecally.

On admittance to the operating theatre, ECG, non-invasive blood pressure, and pulse oximeter will be applied. Basal values will be measured and recorded.

Data collection, including sensory block level, blood pressure, core temperature, and shivering intensity, will be performed every 10 min until the sensory level receded to L4. 


\section{Pre-operative assessment:}

The patients were assessed thoroughly by: Detailed history taking, Full clinical examination. Routine laboratory investigations (complete blood picture, coagulation profile, blood urea, serum creatinine and fasting blood sugar). On arrival to the operating room, patients were connected to:

Multi-channel monitor (Drager
infinity kappa) for monitoring:
Electrocardiogram (ECG). Peripheral pulse oximeter. Non-invasive arterial blood pressure monitor on the dominant arm. External temperature probe. Axillary thermometer.

Anesthetic technique: An intravenous access using 18- Gauge cannula was placed on the non-dominant arm/ hand of all patients. 10 $\mathrm{ml} / \mathrm{kg}$ of intravenous fluids- lactated Ringer's solution- were infused before spinal anesthesia. All fluids were warmed to $37^{\circ} \mathrm{C}$. The ambient temperature was measured by a wall thermometer and maintained at $23^{\circ} \mathrm{C}$. The patients were divided randomly by closed envelope into 2 groups $(n=40)$. Group $R$ received $20 \mathrm{mg}$ of heavy bupivacaine $0.5 \%$ stored at room temperature $\left(23^{\circ} \mathrm{C}\right)$ for 1 hour before time of injection (warm group) plus 25 $\mu \mathrm{g}$ of fentanyl intra-thecally. Group $\mathrm{C}$ received $20 \mathrm{mg}$ of heavy bupivacaine $0.5 \%$ stored at $6^{\circ} \mathrm{C}$ (cold group) plus $25 \mu \mathrm{g}$ of fentanyl intrathecally. The spinal technique was performed with the patient in the sitting position at L3-4 or L4-5 using a $25 \mathrm{G}$ pinpoint spinal needle. Supplemental oxygen $(3 \mathrm{~L} / \mathrm{min})$ was delivered via nasal catheter during the operation. The patient was placed in a supine position. A decrease in the mean blood pressure by $\geq 20 \%$ from baseline value was controlled with i.v ephedrine bolus $5 \mathrm{mg}$ intravenously which was repeated every $1 \mathrm{~min}$. if hypotension persisted or recurred; HR $<50$ beats /min was treated with i.v atropine $0.5 \mathrm{mg}$. The presence of shivering was observed by an observer blinded to the study.

\section{Measurements and recording:}

(1) Haemodynamic parameters: Heart rate in beats per minute. Arterial oxygen saturation. Non-invasive arterial blood pressure (mean blood pressure in $\mathrm{mmHg}$ ).

Timing of haemodynamic parameters: Preoperatively (a baseline value before giving spinal anesthesia and after fluid preload). 0 RESULTS

Table [1]: Comparison between the two studied groups as regards to demographic data minute after giving spinal anesthesia. Every 10 minutes until the end of surgery. Just immediately after the end of the operation and for $0.5 \& 1$ hour postoperatively.

(2) Temperature monitoring: Axillary temperature was recorded with an axillary thermometer. It was measured before intrathecal injection, at 10 min intervals till the end of the operation, just immediately after the end of the operation and for $0.5 \& 1$ hour postoperatively.

(3) Shivering score: It was measured before intra-thecal injection and at $10 \mathrm{~min}$ intervals till the end of the operation and for 1 hour postoperatively.

\section{Statistical analysis of the data ${ }^{(11)}$ :}

Data were fed to the computer and analyzed using IBM SPSS software package version 20.0. (Armonk, NY: IBM Corp). ${ }^{(12)}$ Qualitative data were described using number and percent. The Kolmogorov-Smirnov test was used to verify the normality of distribution Quantitative data were described using range (minimum and maximum), mean, standard deviation and median. Significance of the obtained results was judged at the 5\% level.

\section{The used tests were:}

Chi-square test: For categorical variables, to compare between different groups. Fisher's Exact or Monte Carlo correction: Correction for chi-square when more than $20 \%$ of the cells have expected count less than 5 . Student t-test: For normally distributed quantitative variables, to compare between two studied groups. Paired t-test: For normally distributed quantitative variables, to compare between two periods. ANOVA with repeated measures: For normally distributed quantitative variables, to compare between more than two periods or stages, and Post Hoc test (LSD) for pairwise comparisons. Mann Whitney test: For abnormally distributed quantitative variables, to compare between two studied groups. Wilcoxon signed ranks test: For abnormally distributed quantitative variables, to compare between two periods. Friedman test: For abnormally distributed quantitative variables, to compare between more than two periods or stages and Post Hoc Test (Dunn-Bonferroni) for pairwise comparisons. 
Hassanein Hamzawy et al.

\begin{tabular}{|c|c|c|c|c|}
\hline & Group R & Group C & $\mathbf{t}$ & $\mathbf{p}$ \\
\hline \multicolumn{5}{|l|}{ Age (years) } \\
\hline Range & $22.0-55.0$ & $22.0-54.0$ & \multirow{2}{*}{0.158} & \multirow{2}{*}{0.819} \\
\hline Mean \pm SD & $36.75 \pm 8.08$ & $36.40 \pm 9.02$ & & \\
\hline \multicolumn{5}{|l|}{ Weight (Kgs) } \\
\hline Range & $66.0-101.00$ & $58.00-103.0$ & \multirow[t]{2}{*}{0.670} & \multirow{2}{*}{0.937} \\
\hline Mean \pm SD & $83.42 \pm 9.27$ & $82.12 \pm 10.47$ & & \\
\hline \multicolumn{5}{|l|}{ Height (cms) } \\
\hline Range & $162.0-192.0$ & $159.0-191.0$ & \multirow[t]{2}{*}{0.356} & \multirow{2}{*}{0.051} \\
\hline Mean \pm SD & $176.65 \pm 7.08$ & $176.17 \pm 7.18$ & & \\
\hline
\end{tabular}

$t$ and $p$ : values for Student $t$-test for comparing between the two groups.

Table [2]: Comparison between the two studied groups as regards to heart rate (beats/ min).

\begin{tabular}{|c|c|c|c|c|c|c|c|c|c|c|c|}
\hline & \multicolumn{11}{|c|}{ Heart rate (beats/ minutes) } \\
\hline & \multirow{2}{*}{$\begin{array}{c}\text { Pre- } \\
\text { operative }\end{array}$} & \multicolumn{7}{|c|}{ After giving spinal anaesthesia } & \multirow{2}{*}{$\begin{array}{c}\text { Immediately } \\
\text { after the end } \\
\text { of the } \\
\text { operation }\end{array}$} & \multirow{2}{*}{$\begin{array}{c}0.5 \text { hour } \\
\text { postoperative }\end{array}$} & \multirow{2}{*}{$\begin{array}{l}1 \text { hour } \\
\text { postoperative }\end{array}$} \\
\hline & & $\mathbf{0}$ min & $10 \mathrm{~min}$ & $20 \mathrm{~min}$ & $30 \mathrm{~min}$ & $40 \mathrm{~min}$ & $50 \mathrm{~min}$ & $60 \mathrm{~min}$ & & & \\
\hline \multicolumn{12}{|l|}{ Group R } \\
\hline Min. & 62 & 68 & 67 & 45 & 50 & 60 & 62 & 61 & 61 & 63 & 62 \\
\hline Max. & 118 & 100 & 97 & 88 & 87 & 99 & 102 & 98 & 95 & 94 & 91 \\
\hline $\begin{array}{l}\text { Mean } \\
\pm \text { SD }\end{array}$ & $\begin{array}{l}82.0 \\
\pm 11\end{array}$ & \begin{tabular}{|l|}
80.35 \\
\pm 8.52 \\
\end{tabular} & $\begin{array}{l}79.05 \\
\pm 7.25\end{array}$ & $\begin{array}{l}75.87 \\
\pm 7.91\end{array}$ & $\begin{array}{l}74.17 \\
\pm 7.93\end{array}$ & $\begin{array}{c}75.2 \\
\pm 8.25\end{array}$ & $\begin{array}{l}74.29 \\
\pm 8.6\end{array}$ & $\begin{array}{c}75.0 \\
\pm 8.04\end{array}$ & $\begin{array}{l}75.33 \\
\pm 7.3\end{array}$ & $\begin{array}{l}75.43 \\
\pm 6.77\end{array}$ & $\begin{array}{l}74.95 \\
\pm 7.06\end{array}$ \\
\hline Median & 81.50 & 81.50 & 80.0 & 78.0 & 75.0 & 76.0 & 72.0 & 75.0 & 74.0 & 75.0 & 74.50 \\
\hline $\mathrm{p}_{1}$ & & 0.103 & $0.031^{*}$ & $0.001^{*}$ & \begin{tabular}{|l}
$<0.001^{*}$ \\
\end{tabular} & $0.001^{*}$ & $<0.001^{*}$ & $0.002^{*}$ & $0.001^{*}$ & $0.001^{*}$ & $0.001^{*}$ \\
\hline \multicolumn{12}{|l|}{ Group C } \\
\hline Min. & 60 & 44 & 44 & 45 & 44 & 45 & 55 & 59 & 61 & 62 & 60 \\
\hline Max. & 92 & 95 & 105 & 110 & 112 & 120 & 128 & 89 & 111 & 105 & 99 \\
\hline $\begin{array}{l}\text { Mean } \\
\pm \text { SD }\end{array}$ & $\begin{array}{r}78.62 \\
\pm 8.11 \\
\end{array}$ & $\begin{array}{l}77.32 \\
\pm 9.72 \\
\end{array}$ & \begin{tabular}{|c|}
73.07 \\
\pm 13.08 \\
\end{tabular} & $\begin{array}{c}71.45 \\
\pm \\
14.14 \\
\end{array}$ & $\begin{array}{c}70.32 \\
\pm 12.98 \\
\end{array}$ & $\begin{array}{c}70.25 \\
\pm 12.11 \\
\end{array}$ & $\begin{array}{l}71.05 \\
\pm 12.36\end{array}$ & $\begin{array}{l}69.91 \\
\pm 8.09\end{array}$ & $\begin{array}{l}72.08 \\
\pm 8.65\end{array}$ & $\begin{array}{l}71.75 \\
\pm 7.48\end{array}$ & $\begin{array}{l}70.63 \\
\pm 6.96\end{array}$ \\
\hline Median & 79.0 & 78.50 & 77.0 & 72.0 & 70.0 & 70.0 & 70.50 & 69.0 & 70.50 & 71.0 & 70.0 \\
\hline $\mathrm{p}_{2}$ & & 0.282 & $0.007^{*}$ & $0.001^{*}$ & $<0.001^{*}$ & $<0.001^{*}$ & $0.001^{*}$ & $<0.001^{*}$ & $<0.001^{*}$ & $<0.001^{*}$ & $<0.001^{*}$ \\
\hline$t$ & 1.562 & 1.480 & $2.526^{*}$ & 1.727 & 1.601 & $2.137^{*}$ & 1.325 & $2.485^{*}$ & 1.815 & $2.304^{*}$ & $2.759^{*}$ \\
\hline $\mathrm{p}$ & 0.122 & 0.143 & 0.14 & 0.089 & 0.114 & $0.036^{*}$ & 0.189 & $0.016^{*}$ & 0.073 & $0.024^{*}$ & $0.007^{*}$ \\
\hline
\end{tabular}

$\mathrm{t}, \mathrm{p}$ : $\mathrm{t}$ and $\mathrm{p}$ values for Student t-test for comparing between the two groups.

$\mathrm{p}_{1}$ : $\mathrm{p}$ value for Post Hoc Test (LSD) for ANOVA with repeated measures for comparing between Pre-operatives with each other periods in Group R.

p2: $\mathrm{p}$ value for Post Hoc Test (LSD) for ANOVA with repeated measures for comparing between Pre-operatives with each other periods in Group C.

*: Statistically significant at $\mathrm{p} \leq 0.05$.

Table [3]: Comparison between the two studied groups as regards to peripheral arterial oxygen saturation $\left(\mathrm{SpO}_{2} \%\right)$

\begin{tabular}{|c|c|c|c|c|c|c|c|c|c|c|c|}
\hline & \multicolumn{11}{|c|}{ 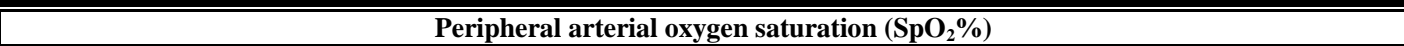 } \\
\hline & \multirow{2}{*}{$\begin{array}{c}\text { Pre- } \\
\text { operative }\end{array}$} & \multicolumn{7}{|c|}{ After giving spinal anaesthesia } & \multirow{2}{*}{$\begin{array}{c}\text { Immediately } \\
\text { after the end } \\
\text { of the } \\
\text { operation }\end{array}$} & \multirow{2}{*}{$\begin{array}{l}0.5 \text { hour } \\
\text { postoperative }\end{array}$} & \multirow{2}{*}{$\begin{array}{c}1 \text { hour } \\
\text { postoperative }\end{array}$} \\
\hline & & $0 \mathrm{~min}$ & $10 \mathrm{~min}$ & $20 \mathrm{~min}$ & $30 \mathrm{~min}$ & $40 \mathrm{~min}$ & $50 \mathrm{~min}$ & $60 \mathrm{~min}$ & & & \\
\hline \multicolumn{12}{|c|}{ 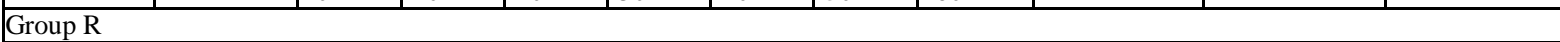 } \\
\hline Min. & 99 & 97 & 97 & 97 & 97 & 97 & 97 & 97 & 97 & 98 & 98 \\
\hline Max. & 100 & 100 & 100 & 100 & 100 & 100 & 100 & 100 & 100 & 100 & 100 \\
\hline $\begin{array}{l}\text { Mean } \\
\pm \text { SD. }\end{array}$ & $\begin{array}{c}99.95 \\
\pm 0.22\end{array}$ & $\begin{array}{l}99.43 \\
\pm 0.73\end{array}$ & $\begin{array}{c}99.08 \\
\pm 0.83\end{array}$ & $\begin{array}{l}98.97 \\
\pm 0.97\end{array}$ & $\begin{array}{l}98.78 \\
\pm 1.05\end{array}$ & $\begin{array}{l}98.73 \\
\pm 0.99\end{array}$ & $\begin{array}{l}98.76 \\
\pm 1.05\end{array}$ & $\begin{array}{l}98.80 \\
\pm 0.96\end{array}$ & $\begin{array}{l}98.98 \\
\pm 0.86\end{array}$ & $\begin{array}{c}99.17 \\
\pm 0.71\end{array}$ & $\begin{array}{l}99.32 \\
\pm 0.69\end{array}$ \\
\hline Median & 100.0 & 100.0 & 99.0 & 99.0 & 99.0 & 99.0 & 99.0 & 99.0 & 99.0 & 99.0 & 99.0 \\
\hline $\mathrm{p}_{1}$ & & $<0.001^{*}$ & $<0.001^{*}$ & $<0.001^{*}$ & $<0.001^{*}$ & $<0.001^{*}$ & $<0.001^{*}$ & $<0.001^{*}$ & $<0.001^{*}$ & $<0.001^{*}$ & $<0.001^{*}$ \\
\hline \multicolumn{12}{|l|}{ Group C } \\
\hline Min. & 99 & 97 & 95 & 95 & 94 & 95 & 95 & 95 & 95 & 97 & 97 \\
\hline Max. & 100 & 100 & 100 & 100 & 100 & 100 & 100 & 100 & 100 & 100 & 100 \\
\hline $\begin{array}{l}\text { Mean } \\
\pm \text { SD. }\end{array}$ & $\begin{array}{l}99.95 \\
\pm 0.22\end{array}$ & $\begin{array}{c}99.0 \\
\pm 0.93\end{array}$ & $\begin{array}{l}98.25 \\
\pm 1.46\end{array}$ & $\begin{array}{l}97.80 \\
\pm 1.56\end{array}$ & $\begin{array}{c}97.83 \\
\pm 1.63\end{array}$ & $\begin{array}{r}97.90 \\
\pm 1.61\end{array}$ & $\begin{array}{l}98.03 \\
\pm 1.46\end{array}$ & $\begin{array}{r}98.09 \\
\pm 1.42\end{array}$ & $\begin{array}{l}98.43 \\
\pm 1.34\end{array}$ & $98.75 \pm 1.08$ & $\begin{array}{l}99.10 \\
\pm 0.87\end{array}$ \\
\hline Median & 100.0 & 99.0 & \begin{tabular}{|l|}
98.0 \\
\end{tabular} & 98.0 & 98.0 & 97.0 & 98.0 & 98.0 & 98.0 & 99.0 & 99.0 \\
\hline $\mathrm{p}_{2}$ & & $<0.001^{*}$ & $\begin{array}{l}<0.001^{*} \\
\end{array}$ & $<0.001^{*}$ & $<0.001^{*}$ & $<0.001^{*}$ & $<0.001^{*}$ & $<0.001^{*}$ & $<0.001^{*}$ & $<0.001^{*}$ & $<0.001^{*}$ \\
\hline $\mathrm{t}$ & 0.00 & $2.248^{*}$ & $3.103^{*}$ & $4.049^{*}$ & $3.097^{*}$ & $2.758^{*}$ & $2.524^{*}$ & $2.303^{*}$ & $2.186^{*}$ & $2.078^{*}$ & 1.278 \\
\hline $\mathrm{p}$ & 1.000 & $0.027^{*}$ & $0.003^{*}$ & $<0.001^{*}$ & $0.003^{*}$ & $0.008^{*}$ & $0.014^{*}$ & $0.025^{*}$ & $0.032^{*}$ & $0.042^{*}$ & 0.205 \\
\hline
\end{tabular}


Table [4]: Comparison between the two studied groups as regards to body temperature $\left(\mathrm{C}^{0}\right)$ using axillary thermometer.

\begin{tabular}{|c|c|c|c|c|c|c|c|c|c|c|c|}
\hline & \multicolumn{11}{|c|}{ Body temperature $\left(\mathrm{C}^{0}\right)$ using axillary thermometer } \\
\hline & \multirow{2}{*}{$\begin{array}{c}\text { Pre- } \\
\text { operativ } \\
\text { e }\end{array}$} & \multicolumn{7}{|c|}{ After giving spinal anaesthesia } & \multirow{2}{*}{$\begin{array}{l}\text { Immediatel } \\
y \text { after the } \\
\text { end of the } \\
\text { operation }\end{array}$} & \multirow{2}{*}{$\begin{array}{l}\text { 0.5 hour } \\
\text { postoperativ } \\
\text { e }\end{array}$} & \multirow{2}{*}{$\begin{array}{c}1 \text { hour } \\
\text { postoperativ } \\
\text { e }\end{array}$} \\
\hline & & $0 \mathrm{~min}$ & $10 \mathrm{~min}$ & $20 \mathrm{~min}$ & $30 \mathrm{~min}$ & $40 \mathrm{~min}$ & $50 \mathrm{~min}$ & $60 \mathrm{~min}$ & & & \\
\hline \multicolumn{12}{|c|}{\begin{tabular}{|l|} 
Group R \\
\end{tabular}} \\
\hline Min. & 37 & 37 & 36.8 & 36.8 & 36.8 & 36.7 & 36.7 & 36.8 & 36.8 & 36.8 & 36.9 \\
\hline Max. & 37.5 & 37.5 & 37.4 & 37.4 & 37.4 & 37.4 & 37.4 & 37.3 & 37.4 & 37.4 & 37.5 \\
\hline $\begin{array}{l}\text { Mean } \\
\pm \text { SD. }\end{array}$ & $\begin{array}{l}37.28 \\
\pm 0.15\end{array}$ & $\begin{array}{l}37.24 \\
\pm 0.15\end{array}$ & \begin{tabular}{|c|}
37.16 \\
\pm 0.15
\end{tabular} & \begin{tabular}{|c|}
37.10 \\
\pm 0.16 \\
\end{tabular} & $\begin{array}{r}37.06 \\
\pm 0.17\end{array}$ & \begin{tabular}{|l|}
37.04 \\
\pm 0.15
\end{tabular} & \begin{tabular}{|c|}
37.03 \\
\pm 0.16
\end{tabular} & \begin{tabular}{|c|}
37.04 \\
\pm 0.14 \\
\end{tabular} & $\begin{array}{c}37.04 \\
\pm 0.14\end{array}$ & $\begin{array}{l}37.05 \\
\pm 0.14\end{array}$ & $\begin{array}{c}37.11 \\
\pm 0.14\end{array}$ \\
\hline \begin{tabular}{|l|} 
Media \\
n \\
\end{tabular} & 37.30 & 37.20 & 37.20 & 37.10 & 37.0 & 37.0 & 37.0 & 37.0 & 37.0 & 37.0 & 37.10 \\
\hline $\mathbf{p}_{1}$ & & $\mathrm{0.001}^{*}$ & $\underset{*}{<0.001}$ & $\underset{*}{<0.001}$ & $\underset{*}{<0.001}$ & $\underset{*}{<0.001}$ & $\underset{*}{<0.001}$ & $\underset{*}{<0.001}$ & $<0.001^{*}$ & $<0.001^{*}$ & $<0.001^{*}$ \\
\hline \multicolumn{12}{|c|}{ Group C } \\
\hline Min. & 37 & 36.8 & 36.6 & 36.5 & 36.5 & 36.5 & 36.5 & 36.6 & 36.5 & 36.6 & 36.8 \\
\hline \begin{tabular}{|l} 
Max. \\
\end{tabular} & 37.8 & 37.8 & 37.8 & 37.5 & 37.5 & 37.5 & 37.4 & 37.4 & 37.4 & 37.5 & 37.5 \\
\hline \begin{tabular}{|l|} 
Mean \\
\pm SD.
\end{tabular} & $\begin{array}{r}37.25 \\
\pm \mathbf{0 . 1 7} \\
\end{array}$ & \begin{tabular}{|c|}
37.18 \\
$\pm \mathbf{0 . 1 9}$ \\
\end{tabular} & \begin{tabular}{|c|}
37.08 \\
$\pm \mathbf{0 . 2 2}$ \\
\end{tabular} & \begin{tabular}{|c|}
36.97 \\
\pm 0.19 \\
\end{tabular} & \begin{tabular}{|l|}
36.93 \\
\pm 0.18 \\
\end{tabular} & \begin{tabular}{|l|}
36.90 \\
\pm 0.18 \\
\end{tabular} & \begin{tabular}{|c|}
36.89 \\
\pm 0.16 \\
\end{tabular} & \begin{tabular}{|c|}
36.92 \\
\pm 0.15 \\
\end{tabular} & $\begin{array}{r}36.91 \\
\pm 0.15 \\
\end{array}$ & $\begin{array}{c}36.97 \\
\pm 0.14 \\
\end{array}$ & $\begin{array}{c}37.02 \\
\pm 0.14 \\
\end{array}$ \\
\hline \begin{tabular}{|l|} 
Media \\
n \\
\end{tabular} & 37.20 & 37.20 & 37.10 & 37.0 & 37.0 & 36.90 & 36.90 & 36.90 & 36.90 & 37.0 & 37.0 \\
\hline $\mathbf{p}_{2}$ & & $\underset{*}{<0.001}$ & $\underset{*}{<0.001}$ & \begin{tabular}{|c|}
$<0.001$ \\
$*$
\end{tabular} & $\underset{*}{<0.001}$ & $\underset{*}{<0.001}$ & $\begin{array}{c}<0.001 \\
*\end{array}$ & $\underset{*}{<0.001}$ & $<0.001^{*}$ & $<0.001^{*}$ & $<0.001^{*}$ \\
\hline$t$ & 0.691 & 1.441 & 1.781 & 3.336* $^{*}$ & $3.366^{*}$ & 3.909* & 3.848* & 3.230* & 3.801* & 2.593* & $2.689^{*}$ \\
\hline $\mathbf{P}$ & 0.492 & 0.153 & 0.079 & $0.001^{*}$ & $0.001^{*}$ & $\begin{array}{c}<0.001 \\
*\end{array}$ & $\underset{*}{<0.001}$ & $0.002^{*}$ & $<0.001^{*}$ & $0.011^{*}$ & $0.009^{*}$ \\
\hline
\end{tabular}

Table [5]: Comparison between the two studied groups as regards to mean arterial blood pressure.

\begin{tabular}{|c|c|c|c|c|c|c|c|c|c|c|c|}
\hline & \multicolumn{11}{|c|}{ Mean arterial blood pressure $(\mathrm{mmHg})$} \\
\hline & \multirow{2}{*}{$\begin{array}{c}\text { Pre- } \\
\text { operative }\end{array}$} & \multicolumn{7}{|c|}{ After giving spinal anaesthesia } & \multirow{2}{*}{\begin{tabular}{|} 
Immediately \\
after the \\
end of the \\
operation
\end{tabular}} & \multirow{2}{*}{$\begin{array}{c}0.5 \text { hour } \\
\text { postoperative }\end{array}$} & \multirow{2}{*}{$\begin{array}{c}1 \text { hour } \\
\text { postoperative }\end{array}$} \\
\hline & & $\begin{array}{c}\mathbf{0} \\
\text { min }\end{array}$ & $10 \mathrm{~min}$ & $20 \mathrm{~min}$ & $30 \mathrm{~min}$ & $40 \mathrm{~min}$ & $50 \mathrm{~min}$ & $60 \mathrm{~min}$ & & & \\
\hline \multicolumn{12}{|c|}{ Group R } \\
\hline Min. & 83.0 & 76.0 & 75.0 & 70.0 & 71.0 & 65.0 & 69.0 & 74.0 & 77.0 & $\mathbf{7 7 . 0}$ & $\mathbf{7 7 . 0}$ \\
\hline Max. & 107.0 & 108.0 & 123.0 & 110.0 & 109.0 & 107.0 & 104.0 & $\mathbf{1 0 0 . 0}$ & 99.0 & 100.0 & 105.0 \\
\hline $\begin{array}{l}\text { Mean } \\
\pm \text { SD }\end{array}$ & $\begin{array}{l}94.47 \\
\pm 6.68\end{array}$ & \begin{tabular}{|l|}
93.63 \\
\pm 6.93 \\
\end{tabular} & $\begin{array}{l}92.78 \\
\pm 8.84\end{array}$ & $\begin{array}{l}89.58 \\
\pm 7.88\end{array}$ & $\begin{array}{l}87.73 \\
\pm 7.19\end{array}$ & $\begin{array}{l}87.40 \\
\pm 7.08\end{array}$ & $\begin{array}{l}87.42 \\
\pm 6.34\end{array}$ & $\begin{array}{l}87.93 \\
\pm 6.53\end{array}$ & $87.90 \pm 5.50$ & $89.15 \pm 5.63$ & $90.05 \pm 6.07$ \\
\hline Median & 95.0 & 94.50 & 93.50 & 90.50 & \begin{tabular}{|l|}
$\mathbf{8 8 . 0}$ \\
\end{tabular} & \begin{tabular}{|l|l|}
88.50 \\
\end{tabular} & 88.0 & \begin{tabular}{|l|}
89.50 \\
\end{tabular} & 88.0 & 90.50 & 90.50 \\
\hline $\mathbf{p}_{1}$ & & 0.786 & 0.365 & $\mathbf{0 . 0 0 1}^{*}$ & $<0.001^{*}$ & $<0.001^{*}$ & $<0.001^{*}$ & $<0.001^{*}$ & $<0.001^{*}$ & $<0.001^{*}$ & $\mathrm{0.001}^{*}$ \\
\hline \multicolumn{12}{|c|}{ Group C } \\
\hline Min. & 94.5 & 66.0 & $\begin{array}{l}57.0 \\
\end{array}$ & 64.0 & 60.0 & 68.0 & 71.0 & 76.0 & 78.0 & $\mathbf{7 7 . 0}$ & 76.0 \\
\hline Max. & 111.0 & 109.0 & 103.0 & 95.0 & 96.0 & 95.0 & 97.0 & 100.0 & 101.0 & 98.0 & 97.0 \\
\hline $\begin{array}{l}\text { Mean } \\
\pm \text { SD } \\
\end{array}$ & $\begin{array}{r}95.47 \\
\pm 6.80 \\
\end{array}$ & \begin{tabular}{|l|}
91.62 \\
\pm 9.77 \\
\end{tabular} & \begin{tabular}{|c|}
86.22 \\
\pm 11.24 \\
\end{tabular} & $\begin{array}{r}83.83 \\
\pm 9.39 \\
\end{array}$ & $\begin{array}{r}83.25 \\
\pm 8.90 \\
\end{array}$ & $\begin{array}{r}83.53 \\
\pm 7.30 \\
\end{array}$ & $\begin{array}{r}85.37 \\
\pm 7.07 \\
\end{array}$ & $\begin{array}{r}87.0 \\
\pm 6.77 \\
\end{array}$ & $87.02 \pm 5.34$ & $\begin{array}{r}86.97 \\
\pm 5.54 \\
\end{array}$ & $87.95 \pm 5.15$ \\
\hline Median & 94.50 & 92.0 & \begin{tabular}{|l|}
88.0 \\
\end{tabular} & 86.0 & \begin{tabular}{|l|}
86.0 \\
\end{tabular} & 85.0 & \begin{tabular}{|l|}
87.0 \\
\end{tabular} & 89.0 & 88.0 & 86.0 & 87.50 \\
\hline $\mathbf{p}_{2}$ & & \begin{tabular}{|l|}
0.062 \\
\end{tabular} & $<0.001^{*}$ & $<0.001 *$ & $<0.001^{*}$ & $<0.001^{*}$ & $<0.001^{*}$ & $<0.001^{*}$ & $<0.001^{*}$ & $<0.001^{*}$ & $<0.001^{*}$ \\
\hline$t$ & 0.663 & \begin{tabular}{|l|}
1.056 \\
\end{tabular} & $2.897^{*}$ & $2.967^{*}$ & $2.473^{*}$ & $2.410^{*}$ & 1.332 & \begin{tabular}{|l|}
0.552 \\
\end{tabular} & $\begin{array}{c}0.721 \\
\end{array}$ & 1.743 & 1.668 \\
\hline p & 0.509 & 0.294 & $\mathrm{0.005}^{*}$ & $0.004 *$ & 0.016* & $\mathbf{0 . 0 1 8}^{*}$ & $\begin{array}{c}\mathbf{0 . 1 8 7} \\
\end{array}$ & $\begin{array}{c}\mathbf{0 . 5 8 3} \\
\end{array}$ & 0.473 & 0.085 & 0.099 \\
\hline
\end{tabular}


Table [6]: Comparison between the two studied groups as regards to shivering score.

\begin{tabular}{|c|c|c|c|c|c|c|c|c|c|c|c|}
\hline & \multicolumn{11}{|c|}{ Shivering score } \\
\hline & \multirow{2}{*}{$\begin{array}{c}\text { Pre- } \\
\text { operative }\end{array}$} & \multicolumn{7}{|c|}{ After giving spinal anaesthesia } & \multirow{2}{*}{$\begin{array}{l}\text { Immediately } \\
\text { after the } \\
\text { end of the } \\
\text { operation }\end{array}$} & \multirow{2}{*}{$\begin{array}{c}0.5 \text { hour } \\
\text { postoperative }\end{array}$} & \multirow{2}{*}{$\begin{array}{c}1 \text { hour } \\
\text { postoperative }\end{array}$} \\
\hline & & 0 min & $10 \mathrm{~min}$ & $20 \mathrm{~min}$ & $30 \mathrm{~min}$ & $40 \mathrm{~min}$ & $50 \mathrm{~min}$ & $60 \mathrm{~min}$ & & & \\
\hline \multicolumn{12}{|c|}{ Group R } \\
\hline $\mathbf{0}$ & $\begin{array}{c}40 \\
(100 \%)\end{array}$ & \begin{tabular}{|c|}
40 \\
$(100 \%)$
\end{tabular} & $\begin{array}{c}28 \\
(70 \%)\end{array}$ & $\begin{array}{c}23 \\
(57.5 \%)\end{array}$ & $\begin{array}{c}17 \\
(42.5 \%) \\
\end{array}$ & $\begin{array}{c}24 \\
(60 \%) \\
\end{array}$ & $\begin{array}{c}18 \\
(45 \%)\end{array}$ & $\begin{array}{c}18 \\
(45 \%)\end{array}$ & $\begin{array}{c}29 \\
(72.5 \%)\end{array}$ & $\begin{array}{c}33 \\
(82.5 \%)\end{array}$ & $\begin{array}{c}37 \\
(92.5 \%)\end{array}$ \\
\hline 1 & $\begin{array}{c}0 \\
(0.0 \%)\end{array}$ & $\begin{array}{c}0 \\
(0.0 \%)\end{array}$ & $\begin{array}{c}12 \\
(30 \%)\end{array}$ & $\begin{array}{c}15 \\
(37.5 \%)\end{array}$ & $\begin{array}{c}18 \\
(45 \%)\end{array}$ & $\begin{array}{c}9 \\
(22.5 \%)\end{array}$ & $\begin{array}{c}15 \\
(37.5 \%)\end{array}$ & $\begin{array}{c}9 \\
(22.5 \%)\end{array}$ & $\begin{array}{c}9 \\
(22.5 \%)\end{array}$ & $\begin{array}{c}7 \\
(17.5 \%)\end{array}$ & $\begin{array}{c}3 \\
(7.5 \%)\end{array}$ \\
\hline 2 & $\begin{array}{c}0 \\
(0.0 \%) \\
\end{array}$ & $\begin{array}{c}0 \\
(0.0 \%) \\
\end{array}$ & $\begin{array}{c}0 \\
(0.0 \%)\end{array}$ & $\begin{array}{c}2 \\
(5 \%) \\
\end{array}$ & $\begin{array}{c}5 \\
(12.5 \%)\end{array}$ & $\begin{array}{c}6 \\
(15 \%) \\
\end{array}$ & $\begin{array}{c}5 \\
(12.5 \%) \\
\end{array}$ & \begin{tabular}{|c|}
3 \\
$(7.5 \%)$ \\
\end{tabular} & $\begin{array}{c}2 \\
(5 \%) \\
\end{array}$ & $\begin{array}{c}\mathbf{0} \\
(\mathbf{0 . 0 \%}) \\
\end{array}$ & $\begin{array}{c}0 \\
(0.0 \%)\end{array}$ \\
\hline 3 & $\begin{array}{c}0 \\
(0.0 \%)\end{array}$ & $\begin{array}{c}0 \\
(0.0 \%)\end{array}$ & $\begin{array}{c}0 \\
(0.0 \%)\end{array}$ & $\begin{array}{c}\mathbf{0} \\
(\mathbf{0 . 0 \%})\end{array}$ & $\begin{array}{c}\mathbf{0} \\
(\mathbf{0 . 0 \%})\end{array}$ & $\begin{array}{c}1 \\
(2.5 \%)\end{array}$ & $\begin{array}{c}\mathbf{0} \\
(\mathbf{0 . 0 \%})\end{array}$ & $\begin{array}{c}\mathbf{0} \\
(\mathbf{0 . 0 \%})\end{array}$ & $\begin{array}{c}0 \\
(0.0 \%)\end{array}$ & $\begin{array}{c}0 \\
(0.0 \%)\end{array}$ & $\begin{array}{c}0 \\
(0.0 \%)\end{array}$ \\
\hline $\begin{array}{c}\text { Median (Min. } \\
- \text { Max.) } \\
\text { Mean } \pm \text { SD. }\end{array}$ & $\begin{array}{c}0.0(0.0- \\
0.0) \\
0.0 \pm 0.0\end{array}$ & $\begin{array}{c}0.0(0.0 \\
-0.0) \\
0.0 \pm \\
0.0\end{array}$ & $\begin{array}{c}0.0(0.0 \\
-1.0) \\
0.30 \pm \\
0.46\end{array}$ & $\begin{array}{c}0.0(0.0 \\
-2.0) \\
0.48 \pm \\
0.60\end{array}$ & $\begin{array}{c}1.0(0.0 \\
-2.0) \\
0.70 \pm \\
0.69\end{array}$ & \begin{tabular}{|c|}
$0.0(0.0$ \\
$-3.0)$ \\
$0.60 \pm$ \\
0.84
\end{tabular} & \begin{tabular}{|c|}
$1.0(0.0$ \\
$-2.0)$ \\
$0.66 \pm$ \\
0.71
\end{tabular} & \begin{tabular}{|c|}
$0.0(0.0$ \\
$-2.0)$ \\
$0.50 \pm$ \\
0.68
\end{tabular} & $\begin{array}{c}\mathbf{0 . 0}(\mathbf{0 . 0}- \\
2.0) \\
0.33 \pm 0.57\end{array}$ & $\left|\begin{array}{c}0.0(0.0-1.0) \\
0.18 \pm 0.38\end{array}\right|$ & {$\left[\begin{array}{c}0.0(0.0-1.0) \\
0.08 \pm 0.27\end{array}\right.$} \\
\hline $\mathbf{p}_{1}$ & & 0.786 & 0.365 & $\mathrm{0.001}^{*}$ & $<0^{\circ .001}{ }^{*}$ & $<0.001^{*}$ & \begin{tabular}{|l|l|}
$<0.001^{*}$ \\
\end{tabular} & $<0.001^{*}$ & $<0.001^{*}$ & $<0.001^{*}$ & $\mathrm{0.001}^{*}$ \\
\hline \multicolumn{12}{|c|}{ Group C } \\
\hline 0 & $\begin{array}{c}40 \\
(100 \%)\end{array}$ & $\begin{array}{c}36 \\
(90 \%)\end{array}$ & $\begin{array}{c}12 \\
(30 \%)\end{array}$ & $\begin{array}{c}6 \\
(15 \%)\end{array}$ & $\begin{array}{c}5 \\
(12.5 \%)\end{array}$ & $\begin{array}{c}5 \\
(12.5 \%)\end{array}$ & \begin{tabular}{|c|}
$\mathbf{3}$ \\
$(\mathbf{7 . 5 \% )}$
\end{tabular} & $\begin{array}{c}7 \\
(17.5 \%)\end{array}$ & $\begin{array}{c}11 \\
(27.5 \%)\end{array}$ & $\begin{array}{c}15 \\
(37.5 \%)\end{array}$ & $\begin{array}{c}32 \\
(80 \%)\end{array}$ \\
\hline 1 & $\begin{array}{c}0 \\
(0.0 \%) \\
\end{array}$ & $\begin{array}{c}4 \\
(10 \%)\end{array}$ & $\begin{array}{c}24 \\
(60 \%)\end{array}$ & $\begin{array}{c}18 \\
(45 \%)\end{array}$ & $\begin{array}{c}12 \\
(30 \%) \\
\end{array}$ & $\begin{array}{c}13 \\
(32.5 \%) \\
\end{array}$ & $\begin{array}{c}15 \\
(37.5 \%) \\
\end{array}$ & $\begin{array}{c}11 \\
(27.5 \%) \\
\end{array}$ & $\begin{array}{c}13 \\
(32.5 \%)\end{array}$ & $\begin{array}{c}24 \\
(60 \%)\end{array}$ & $\begin{array}{c}8 \\
(20 \%) \\
\end{array}$ \\
\hline 2 & $\begin{array}{c}0 \\
(0.0 \%)\end{array}$ & $\begin{array}{c}0 \\
(0.0 \%)\end{array}$ & $\begin{array}{c}4 \\
(10 \%)\end{array}$ & $\begin{array}{c}11 \\
(27.5 \%)\end{array}$ & $\begin{array}{c}12 \\
(30 \%)\end{array}$ & $\begin{array}{c}15 \\
(37.5 \%) \\
\end{array}$ & $\begin{array}{c}19 \\
(47.5 \%)\end{array}$ & $\begin{array}{c}13 \\
(32.5 \%) \\
\end{array}$ & $\begin{array}{c}16 \\
(40 \%)\end{array}$ & $\begin{array}{c}1 \\
(2.5 \%)\end{array}$ & $\begin{array}{c}\mathbf{0} \\
(0.0 \%)\end{array}$ \\
\hline 3 & $\begin{array}{c}\mathbf{0} \\
(\mathbf{0 . 0 \%}) \\
\end{array}$ & $\begin{array}{c}0 \\
(0.0 \%) \\
\end{array}$ & $\begin{array}{c}0 \\
(0.0 \%)\end{array}$ & $\begin{array}{c}5 \\
(12.5 \%) \\
\end{array}$ & $\begin{array}{c}11 \\
(27.5 \%)\end{array}$ & $\begin{array}{c}7 \\
(17.5 \%) \\
\end{array}$ & $\begin{array}{c}1 \\
(2.5 \%) \\
\end{array}$ & $\begin{array}{c}1 \\
(2.5 \%) \\
\end{array}$ & $\begin{array}{c}0 \\
(0.0 \%)\end{array}$ & $\begin{array}{c}\mathbf{0} \\
(\mathbf{0 . 0 \%})\end{array}$ & $\begin{array}{c}0 \\
(0.0 \%)\end{array}$ \\
\hline $\mathbf{p}_{2}$ & & $0.005^{*}$ & $<0.001^{*}$ & $<0.001^{*}$ & $<0^{2001}{ }^{*}$ & $<0.001^{*}$ & \begin{tabular}{|l|l|} 
& \\
\end{tabular} & $<\mathbf{0 . 0 0 1}^{*}$ & $<0.001^{*}$ & $<0.001^{*}$ & $<0.001^{*}$ \\
\hline $\begin{array}{c}\text { Median (Min. } \\
- \text { Max.) } \\
\text { Mean } \pm \text { SD. }\end{array}$ & $\begin{array}{c}0.0(0.0- \\
0.0) \\
0.0 \pm 0.0\end{array}$ & $\begin{array}{c}0.0(1.0 \\
-0.0) \\
0.10 \pm \\
0.30\end{array}$ & $\begin{array}{c}1.0(0.0 \\
-2.0) \\
0.80 \pm \\
0.61\end{array}$ & $\begin{array}{c}1.0(0.0 \\
-3.0) \\
1.38 \pm \\
0.90\end{array}$ & $\begin{array}{c}2.0(0.0 \\
-3.0) \\
1.73 \pm \\
1.01\end{array}$ & \begin{tabular}{|c|}
$2.0(0.0$ \\
$-3.0)$ \\
$1.60 \pm$ \\
0.93
\end{tabular} & \begin{tabular}{|c|}
$2.0(0.0$ \\
$-3.0)$ \\
$1.47 \pm$ \\
0.69
\end{tabular} & $\begin{array}{c}1.0(0.0 \\
-3.0) \\
1.25 \pm \\
0.84\end{array}$ & $\begin{array}{c}1.0(0.0- \\
2.0) \\
1.13 \pm 0.82\end{array}$ & $\begin{array}{c}1.0(0.0-2.0) \\
0.65 \pm 0.53\end{array}$ & {$\left[\begin{array}{c}0.0(0.0-1.0) \\
0.20 \pm 0.41\end{array}\right.$} \\
\hline $\bar{t}$ & 0.663 & 1.056 & $\begin{array}{l}2.897^{*} \\
\end{array}$ & $\begin{array}{l}2.967^{*} \\
\end{array}$ & $\begin{array}{l}2.473^{*} \\
\end{array}$ & $2.410^{*}$ & 1.332 & 0.552 & 0.721 & 1.743 & 1.668 \\
\hline $\mathbf{P}$ & - & 0.116 & $0.001^{*}$ & $<0.001^{*}$ & $<$ o.001 $^{*}$ & $<0.001^{*}$ & $<0.001^{*}$ & $0.004^{*}$ & $<0.001^{*}$ & $<0.001^{*}$ & 0.105 \\
\hline
\end{tabular}

\section{DISCUSSION}

Shivering is an involuntary, oscillatory muscular activity that augments metabolic heat production. Vigorous shivering increases metabolic heat production up to $500-600 \%$ above base level ${ }^{(13)}$.

It is a frequent challenge to anaesthetists as it increases discomfort of the patients and surgeons as regards the mode of anaesthesia. It also interferes with monitoring of electrocardiogram, blood pressure and pulse oxygen saturation. In addition, it increases oxygen consumption, carbon dioxide production and lactic acidosis. It also increases the operative pain by stretching the surgical incision, increase the intraocular pressure and the intracranial pressure. Moreover, it is blamed for hemodynamic changes and increases in heart rate and cardiac output; and thus, it may cause distress to patients especially those with low cardiopulmonary reserve ${ }^{(14)}$.

Shivering, which occurs usually as thermoregulatory response to cold weather may also occur following general or neuraxial anesthesia. Some of the causative factors of this type of shivering may be common to both, but some are particular to neuraxial anesthesia ${ }^{(15)}$.

Shivering is a very common complication related to neuraxial blocks. Postspinal shivering has been reported to occur in $56-63 \%$ of patients undergoing spinal anaesthesia in most of the recent studies ${ }^{(16,17)}$.

It is therefore encouraging to search for some simple and inexpensive interventions and 
medications that are effective in the prevention and treatment of this common as well as serious adverse effect of anaesthesia ${ }^{(18)}$.

The underlying mechanism of postspinal shivering has not been fully established. Yet many efforts have been adopted to overcome the unwanted hypothermia in the perioperative period $^{(13,18)}$.

There are four principal reasons for hypothermia under spinal anaesthesia. Firstly, spinal anaesthesia leads to an internal redistribution of heat from the core to the peripheral compartment. Secondly, with loss of thermoregulatory vasoconstriction below the level of the spinal block, there is increased heat loss from body surfaces. Thirdly, there is altered thermoregulation under spinal anaesthesia characterized by a $0.58^{\circ} \mathrm{C}$ decrease in vasoconstriction and shivering thresholds. Lastly, during either general or regional anaesthesia, a patient's natural behavioral and autonomic responses to cold are unavailable or impaired, and each of general and neuraxial anaesthesia produces high risk for inadvertent perioperative hypothermia ${ }^{(19,20)}$.

Efforts have been made to prevent and treat this serious complication by various methods; such as warming of the operative room and patient, administration of warm intravenous fluids, use of warm and moist gases. In addition, a variety of medications are also used ${ }^{(20)}$.

As regards changes in heart rate, there was no significant difference between the two study groups in the time period immediately before spinal anaesthesia.

In group $\mathrm{R}$ and $\mathrm{C}$, all over the period of follow up after the spinal anaesthesia, there was no significant change in heart rate which may be explained by three reasons. Firstly, the level of the spinal sensory block required had not exceeded T6-7, i.e. there was no affection of the cardiac accelerator fibers which originate from T1-4. Secondly, the patients of the study were given adequate intravenous fluid preloading and intraoperatively, which compensated, to a great extent against the sympathetic blockinduced by the spinal anaesthesia. Lastly, the patients of the study were young adults, ASA III, and with adequate cardiopulmonary reserve.

In the present study, the mean arterial blood pressure (MABP) of the patients in the two studied groups showed no significant statistical differences among the two groups in the time period immediately before spinal anaesthesia.

On comparing the two studied groups statistically, it was found that all over the period of follow up, it was found that there was no significant difference between both groups.

Hypotension occurs as result of block of vasomotor tone which is primarily determined by sympathetic fibers arising from T5 to L1 innervating arterial and venous smooth muscles. Blocking of these nerves causes vasodilatation of the venous capacitance vessels, pooling of blood and decreased venous return to the heart ${ }^{(21)}$. Ten minutes after spinal anaesthesia two patients in group $\mathrm{R}$ developed hypotension for which they received I.V. ephedrine, while there were nine patients in group $\mathrm{C}$ developed hypotension for which they received I.V. ephedrine.

In agreement with the results of this study, Hartmann et al. (22), studied the incidence and risk factors for hypotension after spinal anesthesia induction, by using automated data collection from 3315 patients, hypotension occurred in 166 patients. The analysis identified the following variables to be associated with hypotension after spinal anesthesia: age, weight, height, body mass index, amount of colloid infusion before puncture, amount of bupivacaine $0.5 \%$ used, sensory block height of anesthesia $^{(22)}$.

As regard $\mathrm{S}_{\mathrm{p}} \mathrm{O}_{2}$ of the patients in the present study, there were no statistically significant differences between the two groups.

This constancy of the oxygen saturation within the normal range can be attributed to three reasons. Firstly, the fact that the patients included in the study were ASA III and devoid of any cardiac or pulmonary disorder which could have a reason for oxygen desaturation. Secondly, the use of supplemental oxygen via facemask also helped to maintain the normal oxygen saturation. Lastly, the highest levels of the spinal sensory and motor nerve block have not exceeded T6-7, thus sparing the main respiratory muscle, the diaphragm, which is innervated by $\mathrm{C} 3-5^{(23)}$.

In agreement with the results of this study, Steinbrook et al. ${ }^{(24)}$ studied the effects of spinal anesthesia with bupivacaine on resting pulmonary ventilation and on response to the single breath carbon dioxide test in 11 unpremeditated patients. They concluded that spinal anesthesia was not associated with 
statistically significant changes in tidal volume, respiratory rate, minute ventilation, mean inspiratory flow rate, or the response to the single-breath $\mathrm{CO}_{2}$ test.

Adequate core temperature monitoring is clearly indicated and recommended by the National Institutes for Health and Clinical Excellence's (NICE) Guidelines in all patients undergoing spinal anesthesia for the management of inadvertent perioperative hypothermia and prevention of its serious subsequent complications ${ }^{(25)}$.

In the two study groups, there were no significant differences at the first 10 minutes after spinal anesthesia in axillary temperatures. This may be attributed to the fact that all the patients entered the study being normothermia as a base line for comparison later on.

Twenty minutes after spinal anesthesia, there was significant decrease in axillary temperatures in the two study groups. Later on, all over the time periods of the study, there was significant decrease in axillary temperatures. This may be attributed to three principal reasons for hypothermia under spinal anesthesia. First, spinal anesthesia leads to an internal redistribution of heat from the core to the peripheral compartment, Secondly, with loss of thermoregulatory vasoconstriction below the level of the spinal block; there is increased heat loss from body surfaces. Lastly, there is altered thermoregulation under spinal anesthesia characterized by a $0.5^{\circ} \mathrm{C}$ decrease in vasoconstriction and shivering threshold ${ }^{(18)}$.

In agreement with results of this study, a study by Saito et al. ${ }^{(26)}$ Patients undergoing cesarean delivery were randomly assigned to spinal anesthesia or epidural anesthesia. Spinal anesthesia was induced by injecting $2 \mathrm{~mL} 0.5 \%$ dibucaine into the L4-L5 interspace. Epidural anesthesia was induced with $20 \mathrm{~mL} 2 \%$ mepivacaine injected into the L2-L3 interspace. Thermal comfort and shivering were scored by a blinded observer. They concluded that thermoregulation was impaired more by spinal anesthesia than epidural anesthesia, Tympanic membrane temperatures initially decreased faster during spinal anesthesia, but subsequently decreased at a rate of $\quad 0.5$ degrees $\mathrm{C} / \mathrm{h}$ in both groups.

In a study by Hynson et al. ${ }^{(27)}$ Five healthy, non-pregnant volunteers were studied before and after induction of lumbar epidural anesthesia to determine the cause of central hypothermia during epidural anesthesia. They concluded that central hypothermia during the 1 st hour of epidural anesthesia does not result from heat loss to the environment in excess of metabolic heat production, but results primarily from redistribution of body heat from central to peripheral tissues.

Comparison between the two studied groups showed no significant differences at all the measured times in axillary temperatures.

According to shivering score, in group $\mathrm{R}, 4$ out of 25 patients suffered shivering grade 3 and needed to be given $25 \mathrm{mg}$ pethidine intravenously at 50 minutes after spinal anesthesia. According to the obtained results, warming bupivacaine to 23 -degree $\mathrm{C}$ reduces the incidence and intensity of shivering. This decrease in the incidence of shivering with the group of patients given warm intrathecal bupivacaine in the spinal anesthesia can be attributed to the existence of thermosensory mechanisms in the human spinal canal.

In agreement with this results, study by Mehta et al. $^{(28)}$ A study was undertaken to determine the value of warm local anesthetic and warm parenteral fluids in reducing the incidence of shivering following epidural anesthesia in obstetrics. They concluded that the combined use of warm parenteral fluids and warm local anesthetic significantly reduced the incidence of shivering.

In the current study, In group C, 56\% of patients experienced shivering grade (3), $12 \%$ of patients experienced shivering grade (4) (i.e. 17 out of 25 patients) suffered shivering grades 3 or 4 and needed to be given pethidine intravenously.

Comparison between the two studied groups showed statistically significant differences at all the measured times with increase shivering score at group $\mathrm{C}$.

Ponte $\boldsymbol{e t}$ al. ${ }^{(29)}$ investigates the role of the temperature of the local anesthetic injected extradurally. Forty patients admitted for elective cesarean section under epidural anesthesia were studied; 20 were given bupivacaine warmed to 37 degrees $\mathrm{C}$ (warm group) and 20 were given bupivacaine stored at 4 degrees $\mathrm{C}$ (cold group); the occurrence of shivering in both groups was recorded. The overall incidence of shivering was $27.5 \%$; two patients of the warm group and nine patients of the cold group shivered. This difference was statistically significant ( $\mathrm{P}$ less than 0.03 ). The 
results suggest that there are thermosensory mechanisms in the human spinal canal.

In another study by Walmsley $\boldsymbol{e t}$ al. ${ }^{(30)}$ 30 patients undergoing postpartum tubal ligation under extradural anesthesia initially received bupivacaine at $4^{\circ} \mathrm{C}$, and the incidence of shivering was $47 \%$. Further bupivacaine warmed to $41^{\circ} \mathrm{C}$ was injected into 8 patients in whom the resultant shivering was marked. In 4 patients, the shivering stopped. The authors concluded that thermosensitive tissue within the spinal canal contributes to the shivering observed in association with extradural anesthesia. This finding confirms our findings in this study.

\section{CONCLUSION}

Shivering continues to be a common problem after spinal anesthesia. As shown in this study, the use of warm bupivacaine stored at room temperature $\left(23^{\circ} \mathrm{C}\right)$ for one hour before time of injection into the subarachnoid space can decrease the incidence and intensity of shivering in parturient candidate for elective lower limb orthopedic surgery under spinal anesthesia with no effect on modifying the efficacy of the sensory and motor block or its maximum spread, need for analgesic drugs, pruritus, or vomiting.

\section{REFERENCES}

1. Crowley LJ, Buggy DJ (2008): Shivering and neuraxial anesthesia. Reg Anesth Pain Med., 33:241-52.

2. Piper SN, Fent MT, Röhm KD et al. (2001): Urapidil does not prevent postanesthetic shivering: A dose-ranging study. Can J Anesth., 48:742-7.

3. De Witte J, Sessler DI (2002): Perioperative shivering: Physiology and pharmacology. Anesthesiology, 96:467-84.

4. Doufas AG, Morioka N, Maghoub AN et al. (2008): Lower-body warming mimics the normal epidural-induced reduction in the shivering threshold. Anesth Analg., 106:252-6.

5. Sessler DI, Ponte J (1990): Shivering during epidural anesthesia. Anesthesiology, 72:816-821.

6. Lee GY, Kim CH, Chung RK et al. (2009): Spread of subarachnoid sensory block with hyperbaric bupivacaine. J Clin Anesth., 21:482-485.
7. Van Houwe $P$, Heytens $L$, Vercruysse $P$ (2006): A survey of obstetric an anesthesia practice in Flanders. Acta Anaesth Belg., 57: 29-37.

8. Hawkins JL, Chang J, Palmer SK et al. (2011): Anesthesia-related maternal mortality in the United States: 1979-2002. Obstet Gynecol., 117: 69-74.

9. Alan RA, Graham S, Jonathan T et al. (2013): Smith and Aitkenhead's. Textbook of Anesthesia. Sixth edition Churchill Livingstone, 4: 63-68.

10. Tom EP, Sue H, Mark AW (2008): Pharmacology for Anesthesia and Intensive Care. Third edition Cambridge University.

11. Kotz S, Balakrishnan N, Read CB et al. (2006): Encyclopedia of statistical sciences. 2nd ed. Hoboken, N.J.: WileyInterscience.

12. Kirkpatrick LA, Feeney BC (2013): A simple guide to IBM SPSS statistics for version 20.0. Student ed. Belmont, Calif.: Wadsworth, Cengage Learning.

13. Mathew S, Mulle AL, Verhes PK (2002): Post anesthetic shivering: A new look at tramadol. Anesthesia, 57: 394-8.

14. Hemati K, Baharluie F, Delpisheh A et al. (2015): A randomised clinical trial concerning the effect of treatment with dexamethasone on postoperative shivering. J Anaesth Pain, 4:38-44.

15. Bozgeyik S, Mizrak A, Yendi $F$ et al. (2014): The effects of preemptive tramadol and dexmedetomidine on shivering during arthroscopy. Saudi J Anaesth., 8:238-43.

16. Hendolin H, Lansimies E (1992): Skin and central temperatures during continuous epidural analgesia and general anesthesia in patients subjected to open prostatectomy. Ann Clin Res., 14: 181-6.

17. Frank SM, Beattie C, Christopherson $R$ (1992): Epidural versus general anesthesia, ambient operating room temperature, and patient age as predictors of inadvertent hypothermia. Anesthesiology, 77: 252-7.

18. Matsukawa T, Sessler DI, Christensen $R$ (1995): Heat flow and distribution during epidural anesthesia. Anesthesiology, 83: 961-7.

19. Gordon GG, Goheen MSL, Kenny GP (1997): Inhibition of shivering increases core temperature afterdrop and attenuates rewarming in hypothermic humans. J Appl Physiol., 83: 1630-4. 
20. Wrench IJ, Cavill G, Ward JE, Crossley AWA (1997): Comparison between alfentanil, pethidine and placebo in the treatment of postanaesthetic shivering. Br J Anesth., 79: 541-2

21. Nagan KWD, Khaw KS, Lee BB (2000): A dose response study of prophylactic intravenous ephedrine for prevention of hypotension during spinal anesthesia for cesarean delivery. Anesth Analg., 90: 1390-5.

22. Hartmann B, Junger A, Klasen J (2002): The incidence and risk factors for hypotension after spinal anesthesia induction: An analysis with automated data collection. Anesth Analg., 94:1521-9.

23. Yun E, Topulos GP (1996): Body SC, Pulmonary function changes during epidural anesthesia for cesarean delivery. Anesth Analg., 82(750): 156-181.

24. Steinbrook RA, Concepcion M (1991): Respiratory effects of spinal anesthesia: resting ventilation and single-breath $\mathrm{Co}_{2}$ response. Anesth Analg., 72:182-6.
25. Harper CM, Andrzejowski JC, Alexander R (2008): NICE and warm. $\mathrm{Br}$ J Anesth., 101(3): 293-5.

26. Saito T, Sessler DL, Fujita K et al. (1998): Thermoregulatory effects of spinal and epidural anesthesia during cesarean delivery. Reg Anesth Pain Med., 23:418-23.

27. Hynson JM, Sessler DL, Glosten B et al. (1991): Thermal balance and tremor patterns during epidural anesthesia. Anesthesiology, 74: 680-90.

28. Mehta P, Theriot E, Mehrotra D et al. (1984): Shivering following epidural anesthesia in obstetrics. Reg Anesth Pain Med., 9: 83-5.

29. Ponte J, Sessler DL (1990): Extradurals and shivering: effects of cold and warm extradural saline injections in volunteers. Br J Anaesth., 64: 731-3.

30. Walmsley AJ, Giesecke AH, Lipton JM (1986): Contribution of extradural temperature to shivering during extradural anesthesia. Br J Anaesth., 58:1130-4. 